\title{
Microhabitat characteristics influence shape and size of coral-associated fishes
}

\author{
Florian Wehrberger, Juergen Herler*
}

Department of Integrative Zoology, Faculty of Life Sciences, University of Vienna, Althanstrasse 14, 1090 Vienna, Austria

\begin{abstract}
Coral reefs provide a high diversity of habitats, including the often complex structure of reef-building corals themselves. Since these coral microhabitats are constrained spatially, specific phenotypic adaptations of associated species to the geometric structure of corals are expected. In the northern Red Sea, we investigated how the physical structure of 2 Acropora corals influences the size, shape and growth of fishes that live in these corals. Branch length and interbranch distance increased strongly and differentially with colony size in both coral species, making large colonies more suitable for larger fishes. We used traditional and geometric morphometrics to demonstrate that the 2 coral-dwelling gobies Gobiodon histrio (Valenciennes) and G. rivulatus (Rueppell) follow different ontogenetic growth patterns. Adult G. histrio show a dorsoventrally expanded body combined with a lateral compression, while the wider-bodied G. rivulatus has a smaller maximum body length. The lateral compression enables $G$. histrio to pass smaller interbranch distances than $G$. rivulatus of the same body length, as demonstrated in an experiment testing their ability to pass narrow interbranch distances. Measurements of G. histrio revealed a greater increase of body length and mass with coral colony size in Acropora digitifera than in $A$. gemmifera or other coral species. Outliers in growth rates of the fishes demonstrated that moving to larger corals and/or associating with a larger breeding partner benefits fish growth. Shape-related lateral body size differences provide an explanation why those fishes that are better adapted to certain host corals compete more successfully for these microhabitats. The evolution of different shapes in coral-associated fishes indicates differences in habitat specialization to reef-building corals with different geometries.
\end{abstract}

KEY WORDS: Acropora ' Gobiodon - Coral interbranch-distance - Coral architecture · Adaptation $\cdot$ Phenotype-habitat match $\cdot$ Allometry $\cdot$ Red Sea

\section{INTRODUCTION}

Coral reefs are a complex environment, providing structurally diverse but often spatially constrained microhabitats. Diminution of body size and morphological adaptations have played important roles in coral reef fish evolution (Blake et al. 2005), and small and cryptic species constitute the main proportion of the fishes in modern reefs (Munday \& Jones 1998). These adaptations have enabled them to exploit many small-scaled microhabitats, including the reefbuilding corals themselves. Coral architecture creates variation in the physical environment, providing

\footnotetext{
${ }^{*}$ Corresponding author: juergen.herler@univie.ac.at
}

a broad variety of living space for coral-associated organisms, including the space between coral branches (Munday \& Jones 1998, Herler 2007). Key parameters that physically constrain the size, growth and shape of coral inhabitants are branching form and colony interbranch distance (Munday 2001, Vytopil \& Willis 2001). Interbranch distance, defined as the distance between adjacent branches, represents the space where strictly coral-associated invertebrates and fishes spend most of their lives. This interbranch distance may differ among coral species and may change with colony size, and it is also a physical constraint for the body size and growth of

() The authors 2014. Open Access under Creative Commons by Attribution Licence. Use, distribution and reproduction are unrestricted. Authors and original publication must be credited. 
associated organisms (Sebens 1987, Munday 2001, Vytopil \& Willis 2001, Untersteggaber et al. 2014). As coral-associated fishes are faced with numerous challenges while acquiring food and avoiding predation in their spatially constrained habitat, locomotive performance is crucial for their survival (Fulton 2010). Habitat characteristics often influence the form of inhabiting organisms, and their body shape allows predictions about their maneuverability and locomotion (Webb 1983, Webb et al. 1996, Blake et al. 2005). The form and function of an organism are thus expected to reflect specialization to its preferred microhabitat. Accordingly, coral-associated fishes that live within 3-dimensional structures often evolve a compressed (laterally flattened) body shape, while those living on the surface of massive or flat corals tend to have a highly depressed (dorso-ventrally flattened) body shape (Herler 2007).

Some gobies of the genus Gobiodon have evolved a highly laterally compressed body in adaptation to their lives in Acropora corals, but shapes differ significantly among species (Herler 2007). One of the most compressed species, G. histrio, grows relatively long and exhibits a larger lateral body display compared to congeneric species (Herler \& Hilgers 2005, Herler 2007) but can still move easily among the interbranches of its host coral. G. rivulatus, a space competitor of $G$. histrio, has evolved a different shape to live within the same but also in several other coral species not used by G. histrio (Dirnwöber \& Herler 2007). It has a less compressed body and attains a smaller maximum body length.

Interspecific competition may promote fitnessincreasing phenotypic adaptations toward the preferred coral habitat (Caley \& Munday 2003), and the variety of body shapes is expected to reflect the use of habitats with different geometry (Herler 2007) and/or different levels of specialization (Munday 2001). Common growth patterns are unlikely among fishes, even within closely related species (Klingenberg \& Froese 1991). Ontogenetic trajectories characterize the dynamics of development (Klingenberg 1997), and one hypothesis is that these species follow different ontogenetic trajectories at different rates (Zelditch et al. 2000) during different stages as an adaptation to a life inside corals. To examine whether these 2 gobies exhibit different ontogenetic growth trajectories, we conducted internal shape studies with a focus on the skeleton, using geometric morphometric landmark methods (Bookstein 1997, Mitteröcker \& Gunz 2009). As there is a general sizematch between coral-associated fishes and host corals (Kuwamura et al. 1994, 1996, Hobbs \& Mun- day 2004), we hypothesized that the maximum size (body length and lateral display size) of strictly coralassociated fishes depends on body shape and is constrained by host coral size and architecture. Gobiodon histrio and $G$. rivulatus inhabit colonies of the genus Acropora (Munday et al. 1999). Although they migrate frequently between colonies at early life stages (Wall \& Herler 2009), they spend most of their lives between the branches of their host coral (Feary 2007). Among other Acropora species, they favor the species $A$. digitifera and $A$. gemmifera on shallow reef flats of the Gulf of Aqaba. Untersteggaber et al. (2014) revealed that certain coral parameters such as branch length (BL) or interbranch distance (IBD) determine whether a colony becomes inhabited or not and affect maximum fish size. While BL was the most relevant factor for occupation, IBD is a constraint for the widest dimension of the body (= maximum head width). The question remains how body shape and the underlying skeleton develop in these differently sized fish species under such constraints.

The size, shape and growth of Gobiodon histrio and $G$. rivulatus in relation to the geometry of their most important host corals were examined in a combined approach of morphometric analyses, field observations and laboratory as well as field experiments and addressed the following hypotheses:

(1) The geometry (i.e. IBD and BL) of coral species preferred by habitat-specialized fishes differs from those of less frequently used coral species due to different allometric growth.

(2) The physical constraint of coral IBD governs the maximum size of fishes, which is dependent on fish shape.

(3) Fish size and growth rates depend on coral size, and fishes need to move to larger corals to enhance growth rates and reach their maximum size.

\section{MATERIALS AND METHODS}

\section{Study site and sampling techniques}

All field data were collected in Dahab, Egypt $\left(28^{\circ} 28^{\prime} \mathrm{N}, 34^{\circ} 30^{\prime} \mathrm{E}\right)$, Gulf of Aqaba (northern Red Sea), between October 2010 and May 2011. Fish sampling, coral measurements and experiments were performed in April and May 2011 in a shallow fringing reef ('Napoleon Reef') at a depth of $\sim 1.5 \mathrm{~m}$. The 2 species Gobiodon histrio (larger-sized) and $G$. rivulatus (medium-sized) were selected. All fishes were collected using clove oil, following Munday \& Wilson (1997). Those fishes used for morphometric 
studies were killed by an overdose of clove oil, providing minimal suffering (Munday \& Wilson 1997).

\section{Coral morphometrics}

Measurements were performed on 42 colonies of Acropora digitifera (117 to $1908 \mathrm{~cm}^{2}$ projected planar area) and 22 colonies of $A$. gemmifera (86 to $2591 \mathrm{~cm}^{2}$ projected planar area) because they are the most frequently used host corals by the 2 gobies Gobiodon histrio and $G$. rivulatus in the investigation area. Colony BL, defined as the distance between branch tips and the base (deepest point between 2 main branches), was measured with the depth gauge of a sliding caliper to the closest $1 \mathrm{~mm}$ and repeated for 10 different, randomly chosen branches of each colony. To calculate colony size (in planar projection), the greatest $\left(d_{1}\right)$ and the smallest $\left(d_{2}\right)$ diameter, perpendicular to the former, were measured to the closest cm using a measuring tape. The colony area was then estimated by calculating the area of an ellipse: $d_{1} / 2 \times d_{2} / 2 \times$ pi. To measure the IBD close to branch bases, a new and non-destructive measurement technique employing casts of 2-component epoxy resin (Reef Construct ${ }^{(}$) was used (Untersteggaber et al. 2014). Cylindrical pieces of the mixed resin were individually size-adjusted underwater and then inserted between 2 coral branches with strong stainless steel forceps with graded tips. This process created casts of the lower $1 \mathrm{~cm}$ of interbranch space. Five replicates from representative pairs of main branches (deformed parts of colonies were excluded) were made for each colony and then stored in individually numbered boxes to keep their shape. After the casts had hardened $(\sim 2 \mathrm{~h})$, their width was measured on a fixed height $(7 \mathrm{~mm})$ with a digital sliding caliper to the closest $0.01 \mathrm{~mm}$. The $7 \mathrm{~mm}$ height value equals half of the average head depth of adult specimens of Gobiodon, which is the widest part of the body and considered to be constrained most by interbranch distance (Untersteggaber et al. 2014). Data were processed in Microsoft Excel 2010 for Windows. A 1-way analysis of covariance (ANCOVA) was calculated for IBD measurements and BL across colony size (projected planar area) of both $A$. digitifera and A. gemmifera using the program PAST 2.03.

\section{Fish morphometric analyses}

For morphometric analysis of fishes, we collected a total of 93 juvenile and adult specimens: 61 Gobiodon histrio with total lengths (TL) of 14.9 to $48.3 \mathrm{~mm}$ (specimens $<25 \mathrm{~mm}$ TL were defined as juveniles), and 32 G. rivulatus with TL of 15.4 to $34.7 \mathrm{~mm}$ (specimens $<20 \mathrm{~mm}$ TL were defined as juveniles; Dirnwöber \& Herler 2007). Traditional measurements were made with a digital sliding caliper to the closest $0.01 \mathrm{~mm}$ on ethanol-preserved specimens and included TL (measured from the snout tip to the end of the caudal fin) and greatest head width ( $\mathrm{gHw}$, measured at the widest part of the head, which is at the cheek). All specimens were used to calculate a 1-way ANCOVA for gHw regressed on fish TL to compare speciesspecific growth patterns, using the program PAST 2.03 (Hammer et al. 2001).

To perform geometric morphometric analyses on the skeleton, fishes were cleared and stained with a combination of techniques established by Dingerkus \& Uhler (1977) and Potthoff (1984). To obtain clearer specimens, the eyes and abdominal organs of all specimens were carefully removed. Scanning of cleared and stained fish specimens was done according to Herler et al. (2007), using an EPSON Perfection 4990 Photo flatbed-scanner at 4800 dpi resolution. Each fish was scanned in lateral position in glycerol, and the digital images were processed as follows: on the left side of each specimen, landmarks (homologous points bearing information on the geometry of biological forms; Bookstein 1991) were digitized using the programs tpsUtil 1.46 (Rohlf 2010a) and tpsDig 2.16 (Rohlf 2010b), which generate pixel-based landmark coordinates. To remove non-shape variation, the effects of size, scale and rotation on landmark coordinates of images were removed by a Procrustes superimposition of landmark configurations: the configurations are centered (translation) by their centroid (calculated for each configuration from the arithmetic mean of its landmark coordinates), scaled to a common size by dividing the coordinates of each configuration by its centroid size $(=1$; scaling) and optimally rotated through a least-squares procedure between corresponding landmarks (rotation). The resulting Procrustes shape coordinates can then be analyzed by multivariate statistics. A set of 36 landmarks (Fig. 1) was deployed on the skeletons of 93 specimens. A principal component analysis (PCA) was calculated, and thin-plate-spline deformation grids were created to visualize shape variation along each principal component using the program TPSRelw (Rohlf 2010c). PCA scatter-plots were displayed with the program PAST 2.03 (Hammer et al. 2001). Additionally, a multivariate analysis of variance (MANOVA) was calculated in PAST 2.03 for 


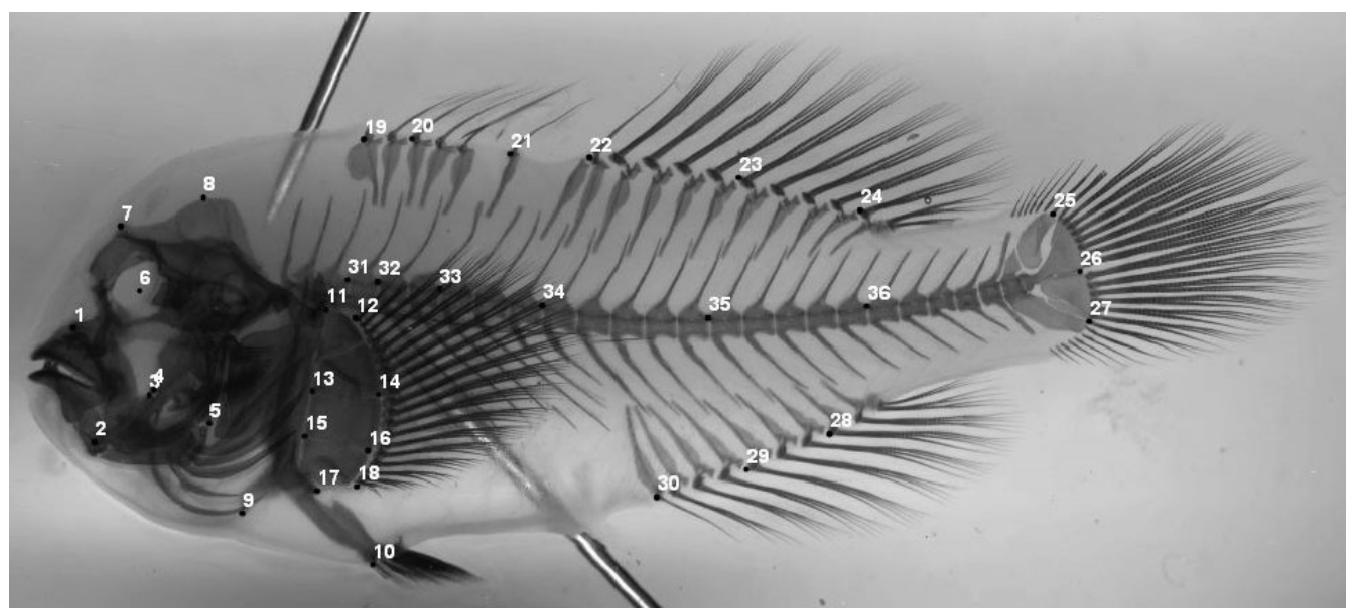

Fig. 1. Position of 36 landmarks for geometric morphometric analysis to capture body shape differences in coral-associated gobiid fishes

the first 10 principal components to check for significant differences between life-stages (juvenile/ adult) and species.

\section{Aquarium experiment: physical constraint of interbranch space}

An aquarium experiment was designed to test the hypothesis that the physical constraint of coral interbranch distance governs the maximum size of fishes. This constraint is expected to depend on fish shape. An artificial model, inspired by Webb et al. (1996), was constructed to simulate coral interbranch distances. It closely resembles the construction used by Kawasaki et al. (2003) but at a different spatial scale. Cylindrical PVC bars, $8 \mathrm{~mm}$ in diameter and $6 \mathrm{~cm}$ high, were fixed onto a $3 \mathrm{~mm}$ thick PVC base. The bars were positioned at different distances and arranged in parallel rows but alternating columns, covering an area of $\sim 20 \times 30 \mathrm{~cm}$. Distances between plastic bars were varied within 6 subdivided fields (each field was $10 \times 10 \mathrm{~cm}$ large) of bars, from a minimum of $3 \mathrm{~mm}$ in the 1st field to a maximum of $7 \mathrm{~mm}$ in the 6th field, resembling the range of interbranch distance found in Acropora digitifera and A. gemmifera. Each field included bar distances that increased at intervals of $0.2 \mathrm{~mm}$ to the next full $\mathrm{mm}$, starting from 0 in the next field. The whole setup was submerged in a small aquarium $(\mathrm{W} \times \mathrm{L} \times \mathrm{H}=$ $25 \times 35 \times 15 \mathrm{~cm}$ ) filled with fresh seawater. Fishes were freshly collected and used only once during experimental trials. A total of 28 Gobiodon histrio (ranging from 29.7 to $48.7 \mathrm{~mm} \mathrm{TL}$ ) and 33 G. rivula- tus (25.4 to $39.9 \mathrm{~mm}$ TL) were used in this experiment. Fishes were acclimatized for $\sim 2 \mathrm{~h}$, put into the aquarium containing the experimental setup, and forced to swim through the variously spaced bars. Fishes were directed toward decreasing bar distances by using a thin plastic stick, but care was taken to ensure that they passed through the bars on their own by merely touching their caudal fin or peduncle laterally. Preliminary experiments revealed that fishes could swim through small interbranch distances in a straight line but could not easily turn around bars at the same distance. Body flexibility was therefore considered to be an important factor (in addition to greatest head width) limiting the locomotion between coral branches. Accordingly, 2 bar distances were measured for each specimen: the minimum distance between 2 bars that was passed in a straight line, and the minimum distance that was passed during a $180^{\circ}$ turn around 1 of 2 adjacent bars. The mean of both values was used for analysis. After each trial, the TL of fishes was measured before they were released back to the reef. Then, $t$-tests were employed to test differences in size between the individuals of the 2 species that passed certain IBDs, and an ANCOVA was calculated to explore differences in size-related swimming abilities between the 2 species.

\section{Long-term field observation: fish size, mass and growth measurements}

These observations were performed to measure general coral size/fish size relationships and to test 
the hypothesis that growth in fishes is dependent on colony size (because of size-related geometry) and enhanced by migration between corals. Between October 2010 and April 2011, size, mass and growth measurements of 55 Gobiodon histrio were carried out in the field. Fishes were collected from host corals in 6 GPS-marked reef plots, each approximately $5 \times$ $5 \mathrm{~m}$ in size. Fishes were taken from their host corals (mostly from Acropora gemmifera and A. digitifera and less frequently from $A$. acuminata, $A$. secale, $A$. samoensis and $A$. valida, combined here as 'others') using a very low dose of clove oil. Host corals were individually tagged, and their maximum diameter (to the closest $1 \mathrm{~cm}$ ) was measured as a proxy for colony size. In the laboratory, fishes were narcotized again with the lowest possible dose of clove oil, scanned on a flatbed scanner (Herler et al. 2007) and damp-dry weighed to the closest $0.1 \mathrm{~g}$. Then, they were tagged with fluorescent elastomer tags (visible implant elastomer [VIE] tags, Northwest Marine Technology) of different colors on 1 of 4 positions on their back to enable individual identification in the field. After recovery overnight in an aquarium with aerated seawater, fishes were taken back to the same host coral they came from. TL was measured from the scans. After 6 mo, fishes were caught again (recapture success was 55 out of 94 tagged specimens $=58 \%$ ), scanned and re-weighed (as performed at their first capture) to determine length and weight increments. Changes of social status (breeding pair or single adult), identity of the breeding partner (tagged or new) and coral colony were noted. Linear regressions and ANCOVAs were calculated for fish body size and mass on coral colony size of each coral species/group. Regressions were also calculated for fish length and mass increments on TL and on body mass, respectively. Two multiple linear regressions were performed in IBM SPSS Statistics 20, one for length increment (in $\mathrm{mm} \mathrm{TL}$ ) and another one for mass increment (in $\mathrm{g}$ ): independent variables were the residuals of the regression of length (in the regression of length increment) and mass (in the regression of mass increment) on colony size (positively correlated in all coral species), change of social status (single adult or breeding pair), change of breeding pair partner, change of coral colony, size difference between old and new coral (if changed) and weight difference between breeding pair partners. In addition, a logistic regression was performed for coral colony change (yes $=1$, no $=0$ ) by employing the independent variables initial body mass, status change and size difference between new and old coral.

\section{Field experiment: fish translocation}

A translocation experiment was performed to experimentally test the idea that physical constraints of coral geometry govern the establishment of particular coral size/fish size relationships. In this experiment, 10 corals were marked and labeled with cable ties and numbers in each of 6 trials. In each trial, 5 large colonies of Acropora digitifera (planar projected area of all large corals ranged from 500 to $1100 \mathrm{~cm}^{2}$ ) and 5 small colonies (with a size range from 120 to $320 \mathrm{~cm}^{2}$ ) were chosen, measured and marked with numbered tags for re-identification. All associated fishes (Gobiodon histrio) were narcotized using clove oil and collected in individually labeled sampling boxes. Then, fishes from the large colonies were transposed into the small colonies and vice versa. As fish and coral size within size groups still varied, gobies were measured for their TL in the field, immediately ranked by size and accordingly assigned to the corals, which were also ranked by size within each size group (e.g. small goby into smallest coral, largest goby into largest coral within the respective coral size group). To re-identify individuals after $1 \mathrm{~d}$, the narcotized specimens were digitally photographed underwater in lateral view. Because of the distinct color patterns of G. histrio, reidentification via images is possible even after long periods of time (Wall \& Herler 2009). Differences in fish size of transposed, emigrated and immigrated fishes were tested with a Mann-Whitney U-test, performed in PAST 2.03.

\section{RESULTS}

\section{Differences in geometry in corals}

A 1-way ANCOVA was calculated for both IBD and BL across colony size (Fig. 2). The increase of interbranch distance with colony size was only slightly greater ( $p=0.045$ for regression slope) in Acropora gemmifera, but the adjusted means were equal ( $p=0.52$ ), indicating a broadly similar IBD. Fig. $2 b$ shows the 1-way ANCOVA of BL against colony area. The adjusted means differed significantly ( $p<0.001)$. Although the regression slopes were not significantly different $(p=0.098)$ because of 2 outliers in $A$. digitifera (slopes become highly significant, $\mathrm{p}=0.006$, when these large but relatively short-branched colonies were removed), ANCOVA clearly shows an overall higher BL of $A$. digitifera colonies. 


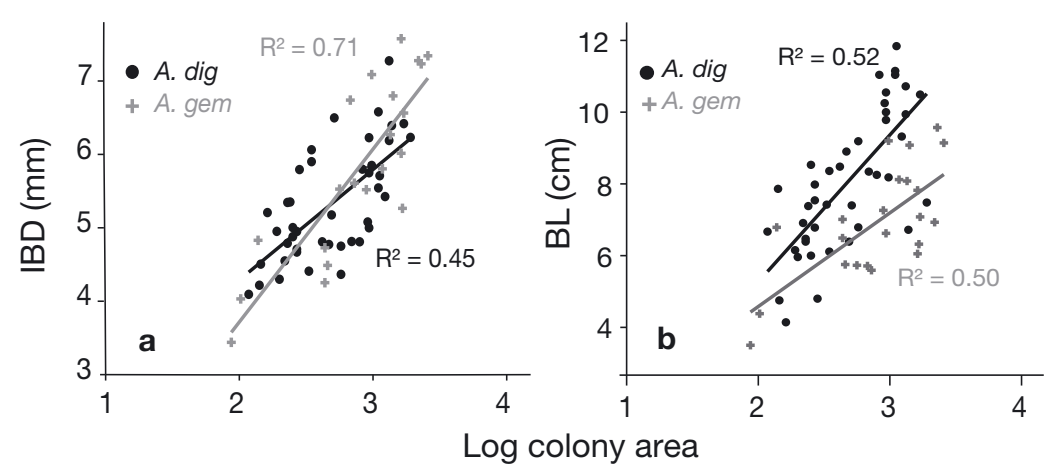

Fig. 2. One-way ANCOVA of colony area $(\log 10)$ and mean values of (a) interbranch distance (IBD) and (b) branch length (BL) from 64 colonies (42 Acropora digitifera, 22 A. gemmifera)

\section{Size and shape differences in fishes}

A 1-way ANCOVA of gHw against fish TL for both Gobiodon histrio and G. rivulatus (Fig. 3) revealed that the slopes and the adjusted means differed significantly $(\mathrm{p}<0.001)$ between the 2 gobies. G. histrio had a greater maximum TL and gHw than G. rivulatus (39 and $13 \%$, respectively), but G. rivulatus had a higher proportional head width.

A PCA of Procrustes shape coordinates of 93 cleared and stained specimens of Gobiodon histrio (21 juveniles, 40 adults) and G. rivulatus (17 juveniles, 15 adults) was calculated, and body shape deformations were visualized via thin-plate-spline deformation-grids (Fig. 4). With proceeding growth, interspecific shape differences increase. G. histrio and G. rivulatus developed into different directions, and the second principal component (PC) separated the 2 species well, in particular adult G. rivulatus

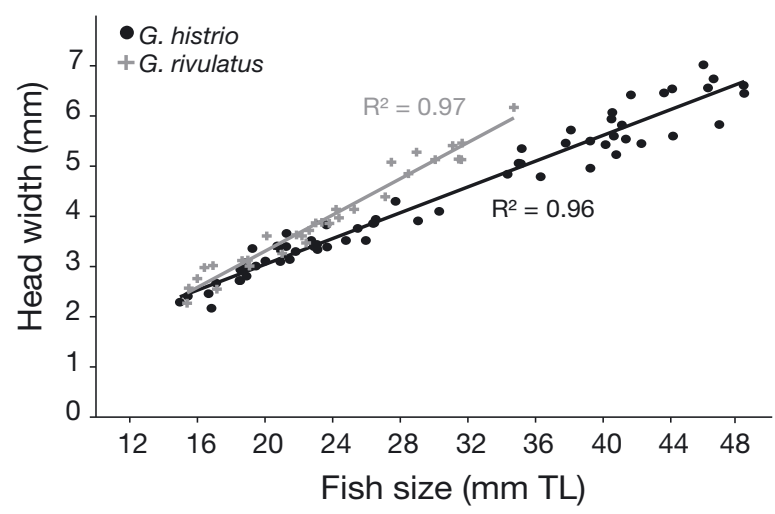

Fig. 3. One-way ANCOVA for greatest head width against total length (TL) in preserved Gobiodon histrio $(\mathrm{n}=61)$ and G. rivulatus $(\mathrm{n}=32)$ from juvenile and adult G. histrio, whereas juvenile G. rivulatus showed an overlap with juvenile $G$. histrio. The ontogenetic shape change of $G$. histrio was mainly expressed along PC 1. The growth trajectory of $G$. rivulatus, by contrast, involved both PCs. This indicates species-specific allometries. As the thin-plate spline deformation grids imply, juvenile specimens had more cylindrical bodies, and there was a strong depression of the anterior body part. The neurocranium section formed a large part of the head region in juvenile specimens. Adult $G$. histrio showed a strong dorso-ventral expansion of the body and underwent a compression of the anterior head region (Fig. 4). Accordingly, regression of PC 1 against centroid size of all specimens showed a significant positive correlation for $G$. histrio $\left(\mathrm{R}^{2}=0.82 ; \mathrm{p}<0.001\right)$ and for G. rivulatus $\left(\mathrm{R}^{2}=0.69 ; \mathrm{p}<0.001\right)$. $\mathrm{PC} 2$ indicated an anterior depression of the body, a pointed head and a more depressed body of G. rivulatus. Regression of PC 2 against centroid size showed no significant correlation for G. histrio $\left(\mathrm{R}^{2}=0.063 ; \mathrm{p}>0.05\right)$ but was highly significant in G. rivulatus $\left(\mathrm{R}^{2}=0.381\right.$; $\mathrm{p}<$ 0.001). A MANOVA on the first 10 principal components (each accounting for at least $1 \%$ of the variance) revealed that differences between juvenile and adult $G$. rivulatus are of relatively low significant difference $(p=0.025)$, whereas all other groups are highly significant different from each other $(\mathrm{p}<$ 0.001; all Bonferroni-corrected).

\section{Fish locomotion in restricted coral interbranch space}

In the aquarium experiment that tested for potential constraints of interbranch space on fish swimming, Gobiodon histrio managed to pass narrower IBDs at the same body size than G. rivulatus, or, in other words, passed the same minimal bar distance at a larger size. The regressions between the minimal bar distance passed and fish body size were significant $(\mathrm{p}<0.001)$, yielded an $\mathrm{R}^{2}>0.90$ in both species (Fig. 5), and were very similar to those of head width against body size (Fig. 3). Even the largest specimens managed to pass an interbranch space of only $5 \mathrm{~mm}$. At this distance, G. rivulatus specimens were $\sim 40 \mathrm{~mm}$ $\mathrm{TL}$, and G. histrio specimens were $48 \mathrm{~mm}$ TL. Thus, among the largest adult fishes that passed the same 


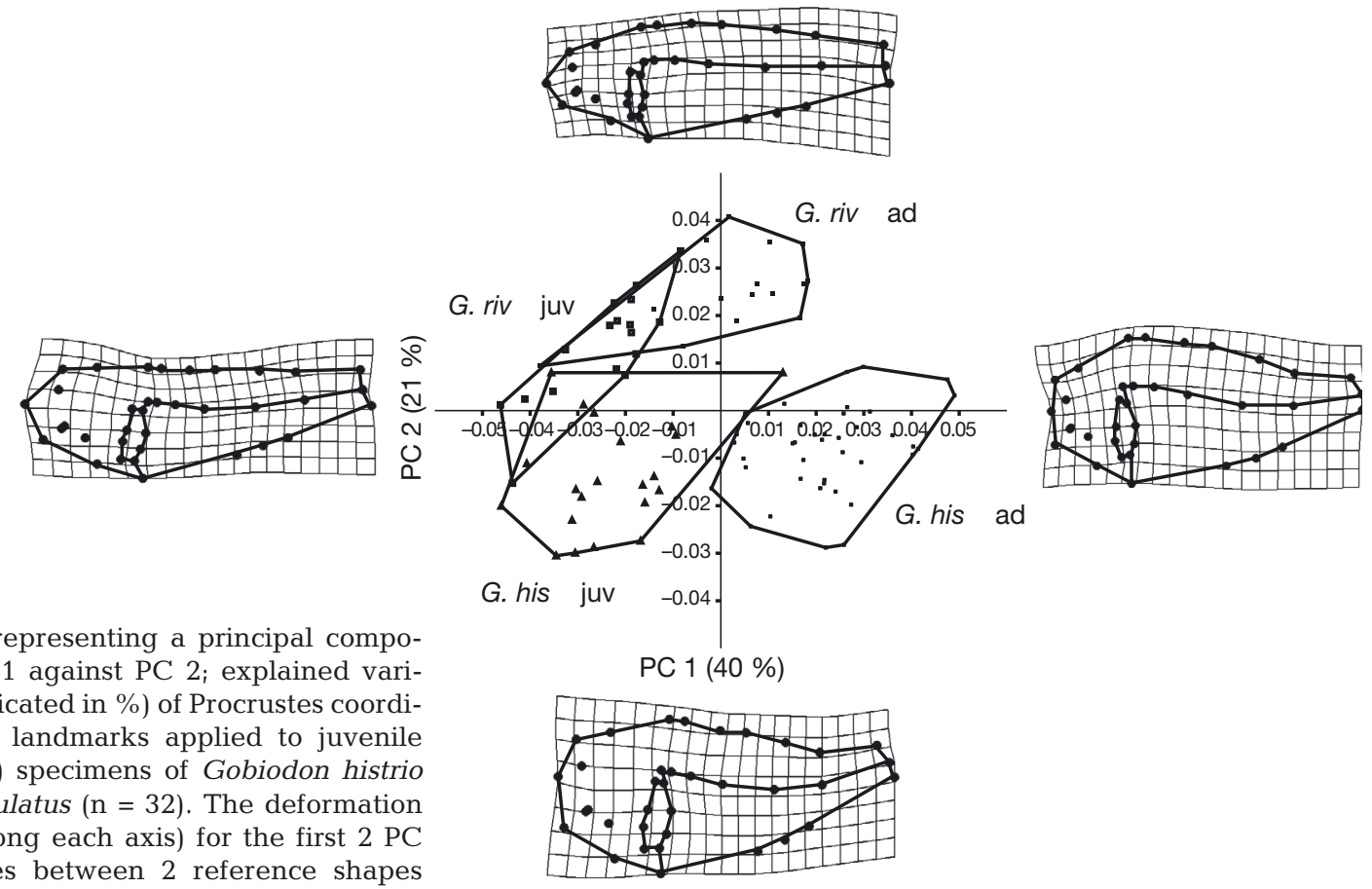

Fig. 4. Scatterplot representing a principal components analysis (PC 1 against PC 2; explained variance of each PC indicated in \%) of Procrustes coordinates based on 36 landmarks applied to juvenile (juv) and adult (ad) specimens of Gobiodon histrio $(\mathrm{n}=61)$ and $G$. rivulatus $(\mathrm{n}=32)$. The deformation grids (0.08 units along each axis) for the first 2 PC visualize differences between 2 reference shapes

bar distance, G. histrio specimens were $20 \%$ longer than G. rivulatus specimens (Fig. 5). G. histrio specimens that had a head that was too wide to pass a bar distance of $<4.5 \mathrm{~mm}$ (the approximately lowest coral interbranch space of inhabited colonies) were significantly larger than G. rivulatus ( $t=9.298, \mathrm{p}<0.001$ ), whereas the minimal bar distances passed by these specimens were the same in both species $(t=0.54$, $\mathrm{p}=0.59$; G. histrio: $\mathrm{n}=10$ and G. rivulatus: $\mathrm{n}=16$ ). A 1-way ANCOVA calculated for the minimal bar

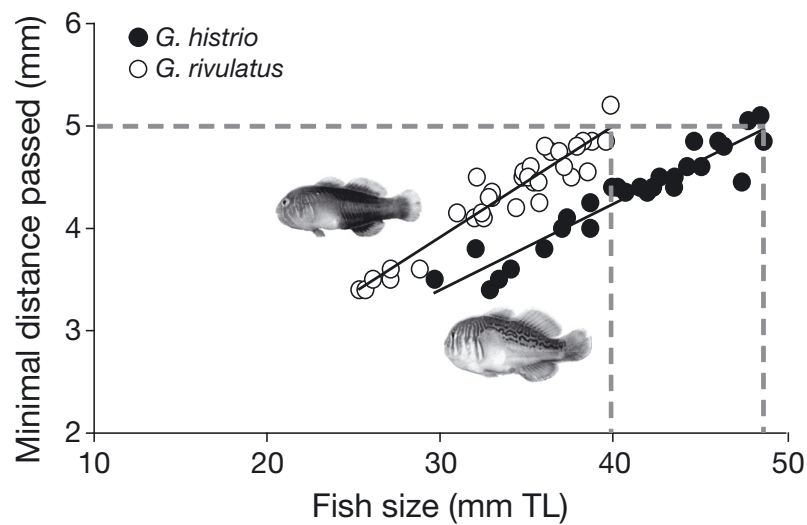

Fig. 5. Regressions of the minimal bar distance (simulated interbranch distance) passed by fishes against their body length (Gobiodon histrio, $\mathrm{n}=42$ and G. rivulatus, $\mathrm{n}=29$ ). Regression lines intersect at $13 \mathrm{~mm}$ total length (TL). Dashed lines indicate the body length difference in $\%$ at a passed bar distance of $5 \mathrm{~mm}$ distance passed by all fishes against their TL revealed a significantly lower $(p<0.001)$ mean bar distance passed by $G$. histrio at the same body size as G. rivulatus.

\section{Coral species and colony-related size, mass and growth of Gobiodon histrio}

In all Acropora species occupied by gobies, fish TL and body mass were significantly positively correlated with colony size $(0.031<\mathrm{p}<0.043$ for length, and $0.008<\mathrm{p}<0.04$ for mass). As revealed by ANCOVA (Fig. 6), fish body mass in regard to coral colony size was significantly higher in A. digitifera than in $A$. gemmifera $(\mathrm{p}<0.05$ for the adjusted mean, $p=0.06$ for the regression slope) or in other Acropora corals (mean: $p<0.001$, slope: $p<0.05$ ). This was similar for fish length, although significant differences were found only between the adjusted means $(\mathrm{p}<0.01)$ of $A$. digitifera and other corals. Absolute fish length increment $\left(\mathrm{mm} \mathrm{mo}^{-1}\right)$ was independent of fish length, whereas there was a weak but significant trend $\left(\mathrm{R}^{2}=0.14, \mathrm{p}=0.004\right)$ that heavier fishes gained more absolute body weight $\left(\mathrm{g} \mathrm{mo} \mathrm{m}^{-1}\right)$ than lighter ones. Relative median body length increment per month was similar in all corals $(0.5,0.4$ and $0.7 \%$ in A. gemmifera, A. digitifera and others, respectively), and the monthly median body mass increment was 
almost equal $(1.8,1.8$ and $2 \%)$. Some rare but interesting growth outliers were found in A. digitifera and A. gemmifera related to the movement of small fishes (single adults) to larger colonies and/or to larger partners. Migration to a larger colony increased monthly growth increments up to 1.8 to $3.7 \%$ (length) and $11 \%$ (mass), association with a larger partner yielded monthly increments of $2.2 \%$ (length) and $7.3 \%$ (mass), and extreme values were attained when both factors were combined $(4.4 \%$ length increment and $18.5 \%$ mass increment, which is $\sim 10$-fold greater than the median). The multiple linear regressions calculated for both length and mass increments considered several of these independent variables: Length increment was significantly positively correlated with colony change $(p=0.027)$ and larger size of the new colony $(p=0.015)$. In contrast, mass increment was only correlated with the residuals of the regression of fish body mass on colony size
( $p<0.01)$; this means that fishes which already were in relatively large corals, compared to their size, gained more mass than others. Change of corals was also important for mass increase but only close to statistical significance $(p=0.068)$. Logistic regression of coral change $(0 / 1)$ revealed that low initial body mass $(\mathrm{p}<0.001)$ and the status change from single adult to breeding pair $(\mathrm{p}=0.03)$ were the most important drivers for changing host colonies.

\section{Fish translocation experiment}

Gobiodon histrio specimens taken from large corals had a mean TL of $4.6 \mathrm{~cm}$ (range $=3.1$ to $5.1 \mathrm{~cm}$, $\mathrm{SD}=0.42$ ), whereas the mean TL of their counterparts, the fishes taken out of small colonies, was $3.3 \mathrm{~cm}$ (range $=2.0$ to $4.6 \mathrm{~cm}, \mathrm{SD}=0.70$ ). A MannWhitney $U$-test showed that the 2 groups differed
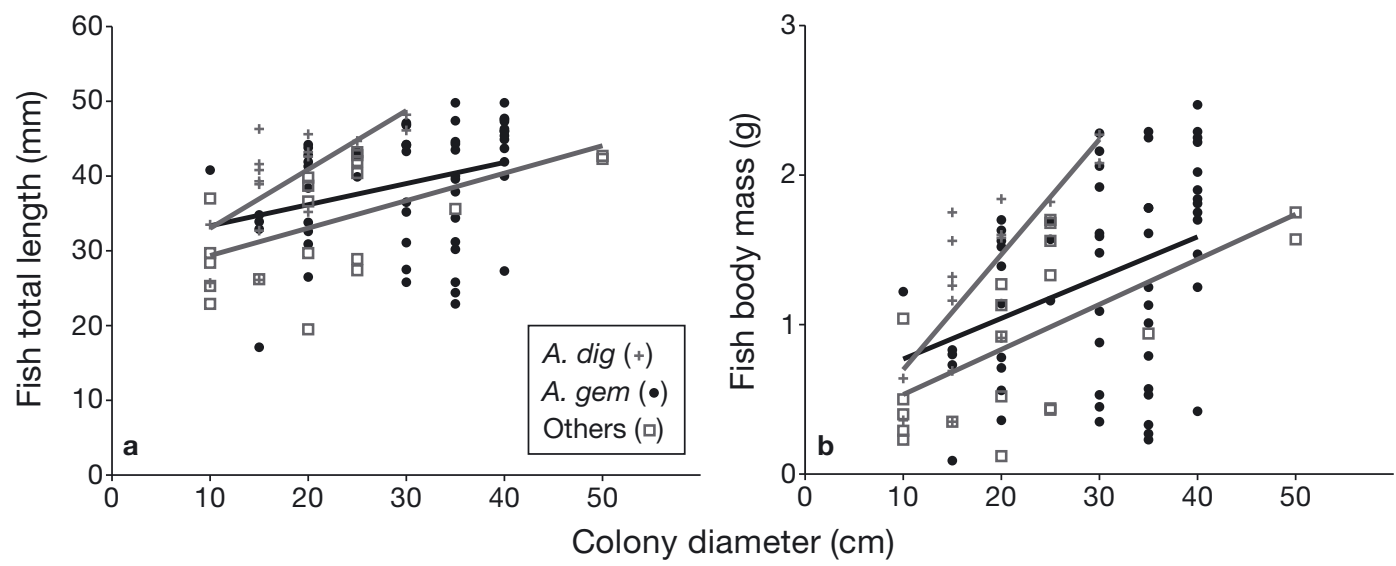

Fig. 6. Gobiodon histrio. (a) Size (body length) and (b) mass of G. histrio regressed on colony size in 3 different coral species/ groups (Acropora digitifera, A. gemmifera, and A. acuminata; A. secale, A. samoensis and A. valida combined as 'others')
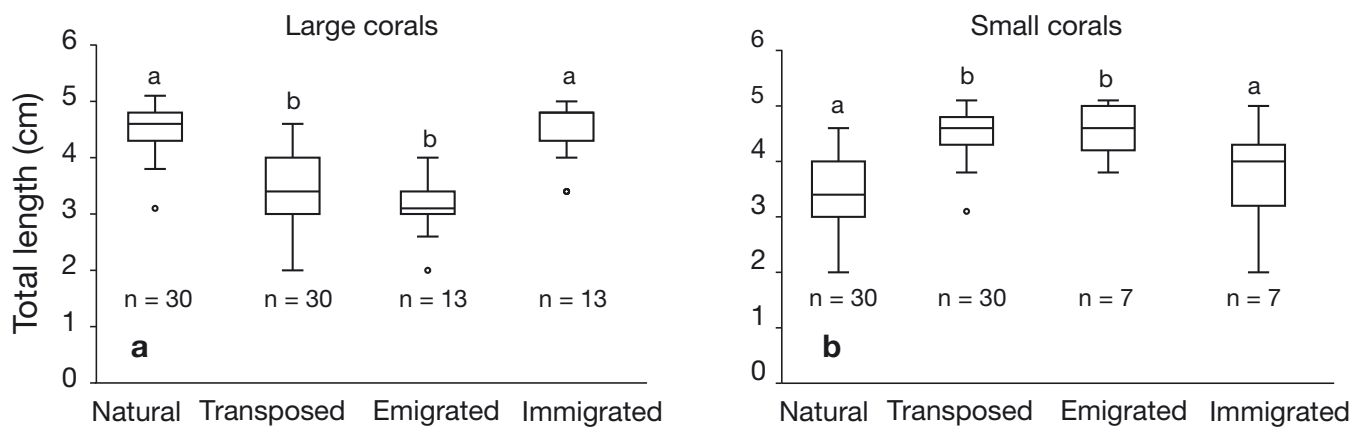

Fig. 7. Size ranges of fishes from (a) large and (b) small corals in the translocation experiment. The first 2 boxes represent fishes taken out from one coral size class and transposed into the other size class, and vice versa (explaining the same plots in switched positions in a and b). The third box-plot shows the size of fishes that left the corals after $24 \mathrm{~h}$ (emigrated), and the fourth plot represents fishes that moved in (immigrated). Box plots show the median (horizontal line), the upper and lower quartiles, the ranges (whiskers) and outliers (dots). Significant differences $(p<0.001)$ of groups are marked by different letters $(\mathrm{a}, \mathrm{b})$ above the plots. Numbers under plots indicate number of fishes/corals 
significantly from each other in TL $(p<0.001)$. One day after translocation, 13 (43\%) of the 30 smaller fishes (mean TL $=3.2 \mathrm{~cm}$ ) had abandoned the large colonies, while much larger specimens (mean TL = $4.5 \mathrm{~cm}$ ) moved in (Fig. 7a). Six colonies (20\%) were found empty after $1 \mathrm{~d}$, and in 11 (53\%) corals, the transposed fish remained. A trend in the other direction was observed in small colonies (Fig. 7b). After $1 \mathrm{~d}, 7(23 \%)$ of the 30 transposed larger fish (mean $\mathrm{TL}=4.5 \mathrm{~cm}$ ) had abandoned the small corals, and 7 small- to medium-sized fish (mean TL $=3.8$ ) had moved in. Additionally, 7 (23\%) colonies were found empty, and in about half of the colonies $(n=16$, mean fish TL = 4.6), the transposed fish remained. A MannWhitney $U$-test showed that immigrants differed from transposed and emigrated fishes ( $\mathrm{p}<0.001$; Bonferroni-corrected) but equalled the size of the original ones (e.g. there was no significant difference between immigrated and original specimens: $\mathrm{p}=0.1$ in small and $\mathrm{p}=0.99$ in large colonies).

\section{DISCUSSION}

In the present study, we investigated the effect of host coral size and growth-related changes of coral architecture on the size, shape and growth of associated gobiid fishes. Measurements of the development of IBD and BL in corals should reveal coral-sizedependent spatial constraints for fish inhabitants. The growth patterns of 2 gobiids of the genus Gobiodon, which show similar habitat demands in the northern Red Sea but differ in habitat specialization, body shape and maximum body size, were examined by means of geometric morphometrics of their skeleton. An aquarium experiment examined the potential of specialized body shapes for attaining a greater body size within the spatially constrained coral IBD. A long-term observation of tagged fishes was carried out to examine the coral traits that promote larger body size and mass and higher growth rates in fishes, and a transplantation experiment was designed to show that size-dependent coral architecture actually puts selection pressure on the size of associated fishes in the field.

\section{Growth patterns and shape differences in corals and fishes}

Coral shape variables such as IBD or BL show different ontogenetic development in Acropora digitifera and A. gemmifera. Both variables increase with colony size, making large colonies more suitable for larger fishes (Hobbs \& Munday 2004, Kuwamura et al. 1994) and thus for breeding pairs of Gobiodon (Wall \& Herler 2009). Comparing the 2 coral species, A. digitifera clearly provides better host coral qualities. In similar-sized colonies, branches are longer than in $A$. gemmifera while IBDs are similar. These 2 factors combined offer better protection against predation, also explaining the high occupation rate (up to $86 \%$ ) of $A$. digitifera colonies in the investigation area (Schiemer et al. 2009). Untersteggaber et al. (2014) discovered that the mean BLs of corals occupied by adult fishes was greater $(>5 \mathrm{~cm}$ ) than in unoccupied colonies. Although narrow-branched corals support a greater abundance of organisms (Vytopil \& Willis 2001), IBD seems to limit maximum size and growth rate in fishes (Munday 2001). This enforces shape adaptation in obligatorily coral-associated fishes. Apart from IBD, colony area is significantly correlated with fish TL, indicating a size match between host and fish (Kuwamura et al. 1994, Hobbs \& Munday 2004).

Gobiodon histrio and G. rivulatus differ significantly in size and shape, and the shape differences between the species increase with growth. Both species were similarly shaped up to $\sim 24 \mathrm{~mm} \mathrm{TL}$, but then, different allometries become noticeable. $G$. rivulatus develops a wider head and body, accompanied by a shorter maximum TL. Both geometric and traditional morphometrics revealed that $G$. histrio shows a dorso-ventrally expanded and compressed body and a smaller relative head width, which represents the widest part of the body.

\section{Ecological consequences of different body form}

More compressed species, such as Gobiodon histrio, can move more easily through the restricted space between coral branches, as revealed in an aquarium experiment. IBD and fish head width had a similar range, but fishes can squeeze through narrower gaps than head width measurements suggest. For optimal locomotion, however, IBD should be equal to or larger than the head width of its fish inhabitant (Untersteggaber et al. 2014). Thus, IBD clearly sets a limit for maximum fish size, depending on the species' shape. Restricted body size has also been observed in other organisms that occupy the interbranch space of corals. Vytopil \& Willis (2001) found smaller Tetralia crabs in Acropora hyacinthus than in A. millepora, which has a larger interbranch area, and in Gobiodon spp., different growth and survival rates are related to differences in the interbranch space (Munday 2001). 
According to the translocation experiment and the long-term field measurements of fish body size and mass, fishes can grow larger within long-branched coral species (Acropora digitifera in the present study) and large colonies. A large fish body size leads to a higher rank in the competitive hierarchy and permits the occupation of the best habitat patches (Holbrook \& Schmitt 2002, Hobbs \& Munday 2004). Colony size is an important life-history characteristic of corals (McClanahan et al. 2008) and has an effect on the inhabiting organisms. Larger colonies generally supported larger fishes, but coral size alone did not determine host quality. In addition, certain geometric features such as BL and IBD are important for fish occupation and size. As Untersteggaber et al. (2014) have shown, short-branched corals ( $<5 \mathrm{~cm} \mathrm{BL)}$ are less frequently occupied. This may reflect better access of predators to short-branched colonies and high selective pressure against the choice of such corals by gobies. Because high-quality host colonies are limited, vacant space is rapidly colonized (Munday et al. 1997, 2001), and fishes may be forced to live in suboptimal corals.

In addition to interspecific competition, intraspecific competition may play an important role in the distribution of coral-dwelling fishes (Munday 2000, 2001, Holbrook \& Schmitt 2002, Hobbs \& Munday 2004). The manipulative translocation experiment, in which large fishes were transposed to small colonies and vice versa, clearly exceeded natural thresholds between colony size and fish size. This led to high emigration and immigration rates in both small and large colonies soon after translocation. It was not investigated whether fishes abandoned the corals they were transposed to because they preferred more favorably sized corals or simply because they did not accept the removal from the original host colony. Nevertheless, as small gobies also favor large colonies over smaller ones (Hobbs \& Munday 2004), they should accept translocation to larger colonies, and it is most likely that many of the small fishes were forced out of the large colonies by larger conspecifics rather than leaving their colonies voluntarily: more large colonies ( $43 \%$ ) had larger fishes than were empty (20\%) after $1 \mathrm{~d}$. In contrast, large gobies require a minimum IBD accompanied by a certain colony area to gain shelter against predators and sufficient breeding space (Kuwamura et al. 1996, Untersteggaber et al. 2014), and we assume that the large fishes that abandoned the small colonies did so mainly because of insufficient space (the proportion of colonies that were empty and those which had smaller fishes was equal, $23 \%$ ). This indicates that availability of large corals is a more important determinant for fish distribution than the physical restriction by coral size. Based on a significant correlation between fish length and coral size (Hixon \& Beets 1989, Kuwamura et al. 1994, Patton 1994, Friedlander \& Parrish 1998, and the present study), the size and growth of gobies are apparently restricted by the architecture and size of their host corals. Interestingly, extreme growth rates were attained by smaller fishes when they managed to move to larger colonies and/or become associated with a large partner. This conforms with strategic growth regulation that has been described in coral-dwelling gobies (Munday et al. 2006, Wong et al. 2007, 2008). There is certainly a trade-off between the lower risk of predation by avoiding movement between colonies and a lower growth rate in less optimal corals.

Strong competition for habitats may be the main reason why species such as Gobiodon histrio evolved such an extremely compressed body shape: the larger overall body size helps achieve a higher rank in a size-based competitive hierarchy. Moreover, fish length does not implicitly reflect the fish's 'functional body size' (Wiederholm 1987). The lateral compression combined with the dorso-ventrally expanded body results in a particularly larger lateral body display (Untersteggaber et al. 2014), and this is important during turf wars (Collyer et al. 2005). Also, individual fitness-related advantages among distinct coral species may explain why $G$. histrio is perfectly adapted to its host corals and competes strongly for them (Munday 2000). This growth pattern can be seen as an adaption to its preferred habitat, allowing this specialist to grow faster (Caley \& Munday 2003) and larger (Herler 2007) in its preferred host coral. This results in a fitness advantage in this space-limited microhabitat over morphologically less welladapted species (Puebla 2009). Extreme body shapes, however, may trade off with physiological functions of organs or locomotory abilities (Webb 1984, Hanken \& Wake 1993). A strongly compressed body may be advantageous only in certain microhabitats and is thus reflected in high microhabitat specialization, which provides an explanation why not all species of the genus Gobiodon have evolved such specialized body shapes.

Apart from improving our understanding of factors governing the habitat preferences and resource partitioning among coral-associated fishes, the present study may help to make predictions about the consequences of coral loss and changes in coral composition (Hoegh-Guldberg et al. 2007, Hughes et al. 2007) and in colony size (McClanahan et al. 2008) for 
associated organisms in future coral reefs. The diversity and structural complexity of coral microhabitats may become less as numerous factors such as coral bleaching (Baird \& Marshall 2002, Wilkinson 2004) or crown-of-thorns seastar outbreaks (Moran et al. 1992, Lourey et al. 2000) degrade and destroy coral reefs. Habitat loss will increase inter- and intraspecific competition for remaining corals, and a smaller proportion of large colonies within coral communities will negatively affect large adult gobies and especially breeding pairs, which show the lowest movement rate between colonies (Wall \& Herler 2009). Additionally, smaller body size at maturity will be advantageous in a reef dominated by smaller colonies, and larger-growing species will be forced to mature earlier and remain smaller, which would change competitive hierarchies. Increased environmental stress will thus certainly affect the abundance and diversity of coral-associated fishes, not only because of habitat loss but also by altering the habitat structure.

Acknowledgements. We are grateful to the Nature Conservation Sector of the Egyptian Environmental Affairs Agency (NCS/EEAA; Moustafa Fouda) for granting research and sampling permissions and to the Suez Canal University, Egypt (Magdy El-Alwany), for co-operation. A. Wanninger (Department of Integrative Zoology, University of Vienna) provided workspace and laboratories at the University of Vienna. The Dahab Marine Research Center (DMRC; A. Tischer ${ }^{\Psi}$ and H. Lange) supported us with field laboratories and diving logistics. We thank M. Dirnwöber for his support during the fieldwork in Dahab and M. Stachowitsch for linguistic corrections. P. Mitteröcker and I. Zweimüller assisted with statistics. This study was financed by the Austrian Science Fund (FWF; project number P21616-B12).

\section{LITERATURE CITED}

Baird AH, Marshall PA (2002) Mortality, growth and reproduction in scleractinian corals following bleaching on the Great Barrier Reef. Mar Ecol Prog Ser 237:133-141

Blake RW, Law TC, Chan KHS, Li JFZ (2005) Comparison of the prolonged swimming performances of closely related, morphologically distinct three-spined sticklebacks Gasterosteus spp. J Fish Biol 67:834-848

Bookstein FL (1991) Morphometric tools for landmark data: geometry and biology. Cambridge University Press, New York, NY

> Bookstein FL (1997) Landmark methods for forms without landmarks: morphometrics of group differences in outline shape. Med Image Anal 1:225-243

> Caley JM, Munday PL (2003) Growth trades off with habitat specialization. Proc R Soc Lond 270:S175-S177

Collyer ML, Novak JM, Stockwell CA (2005) Morphological divergence of native and recently established populations of white sands pupfish (Cyprinodon tularosa). Copeia 2005:1-11
Dingerkus G, Uhler LD (1977) Enzyme clearing of alcian blue stained whole small vertebrates for demonstration of cartilage. Stain Technol 52:229-232

> Dirnwöber M, Herler J (2007) Microhabitat specialisation and ecological consequences for coral gobies of the genus Gobiodon in the Gulf of Aqaba, northern Red Sea. Mar Ecol Prog Ser 342:265-275

> Feary DA (2007) The influence of resource specialization on the response of reef fish to coral disturbance. Mar Biol 153:153-161

Friedlander AM, Parrish JD (1998) Habitat characteristics affecting fish assemblages on a Hawaiian coral reef. J Exp Mar Biol Ecol 224:1-30

Fulton CJ (2010) The role of swimming in reef fish ecology. In: Domenici P, Kapoor BG (eds) Fish swimming: an ethoecological perspective. Science Publishers, Enfield

Hammer Ø, Harper DAT, Ryan PD (2001) PAST: palaeontological statistics software package for education and data analysis. Palaeontol Electronica 4:9

> Hanken J, Wake DB (1993) Miniaturization of body size: organismal consequences and evolutionary significance. Annu Rev Ecol Syst 24:501-519

> Herler J (2007) Microhabitats and ecomorphology of coraland coral rock-associated gobiid fish (Teleostei: Gobiidae) in the northern Red Sea. Mar Ecol 28:82-94

Herler J, Hilgers H (2005) A synopsis of coral and coral-rock associated gobies (Pisces: Gobiidae) in the Gulf of Aqaba, northern Red Sea. Aqua J Ichthyol Aquat Biol 10: 103-132

Herler J, Lipej L, Makovec T (2007) A simple technique for digital imaging of live and preserved small fish specimens. Cybium 31:39-44

Hixon MA, Beets JP (1989) Shelter characteristics and Caribbean fish assemblages: experiments with artificial reefs. Bull Mar Sci 44:666-680

> Hobbs JPA, Munday PL (2004) Intraspecific competition controls spatial distribution and social organisation of the coral-dwelling goby Gobiodon histrio. Mar Ecol Prog Ser 278:253-259

Hoegh-Guldberg O, Mumby PJ, Hooten AJ, Steneck RS and others (2007) Coral reefs under rapid climate change and ocean acidification. Science 318:1737-1742

> Holbrook SJ, Schmitt RJ (2002) Competition for shelter space causes density-dependent predation mortality in damselfishes. Ecology 83:2855-2868

- Hughes TP, Rodrigues MJ, Bellwood DR, Ceccarelli D and others (2007) Phase shifts, herbivory, and the resilience of coral reefs to climate change. Curr Biol 17:360-365

Kawasaki H, Sano M, Shibuno T (2003) The relationship between habitat physical complexity and recruitment of the coral reef damselfish, Pomacentrus amboinensis: an experimental study using small-scale artificial reefs. Ichthyol Res 50:73-77

Klingenberg CP (1997) Heterochromy and allometry: the analysis of evolutionary change in ontogeny. Biol Rev Camb Philos Soc 73:79-123

Klingenberg CP, Froese R (1991) A multivariate comparison of allometric growth patterns. Syst Biol 40:410-419

Kuwamura T, Yogo Y, Nakashima Y (1994) Population dynamics of goby Paragobiodon echinocephalus and host coral Stylophora pistillata. Mar Ecol Prog Ser 103:17-23

Kuwamura T, Nakashima Y, Yogo Y (1996) Plasticity in size and age at maturity in a monogamous fish: effect of host coral size and frequency dependence. Behav Ecol Sociobiol 38:365-370 
Lourey MJ, Ryan AJD, Miller IR (2000) Rates of decline and recovery of coral cover on reefs impacted by, recovering from and unaffected by crown-of-thorns starfish Acanthaster planci: a regional perspective of the Great Barrier Reef. Mar Ecol Prog Ser 196:179-186

McClanahan TR, Ateweberhan M, Omukoto J (2008) Longterm changes in coral colony size distributions on Kenyan reefs under different management regimes and across the 1998 bleaching event. Mar Biol 153:755-768

Mitteröcker P, Gunz P (2009) Advances in geometric morphometrics. Evol Biol 36:235-247

Moran PJ, De'ath G, Baker VJ, Bass DK and others (1992) Pattern of outbreaks of crown-of-thorns starfish (Acanthaster planci L.) along the Great Barrier Reef since 1986. Aust J Mar Freshw Res 43:555-567

Munday PJ (2000) Interactions between habitat use and patterns of abundance in coral-dwelling fishes of the genus Gobiodon. Environ Biol Fishes 58:355-369

Munday PL (2001) Fitness consequences of habitat use and competition among coral-dwelling fishes. Oecologia 128: 585-593

Munday PL, Jones GP (1998) The ecological implications of small body size among coral-reef fishes. Oceanogr Mar Biol Annu Rev 36:373-411

Munday PL, Wilson SK (1997) Comparative efficacy of clove oil and other chemicals in anaesthetization of Pomacentrus amboinensis, a coral reef fish. J Fish Biol 51:931-938

> Munday PL, Jones GP, Caley MJ (1997) Habitat specialisation and the distribution and abundance of coraldwelling gobies. Mar Ecol Prog Ser 152:227-239

Munday PL, Harold AS, Winterbottom R (1999) Guide to coral-dwelling gobies (genus Gobiodon) of Papua New Guinea and the Great Barrier Reef. Rev Fr Aquariol 26: 53-58

Munday PL, Jones GP, Caley MJ (2001) Interspecific competition and coexistence in a guild of coral-dwelling fishes. Ecology 82:2177-2189

Munday PL, Cardoni AM, Syms C (2006) Cooperative growth regulation in coral-dwelling fishes. Biol Lett 2: 355-358

Patton WK (1994) Distribution and ecology of animals associated with branching corals (Acropora spp.) from the Great Barrier Reef, Australia. Bull Mar Sci 55:193-211

Potthoff T (1984) Clearing and staining techniques. Ontogeny and systematics of fishes. In: Moser HG (ed) American Society of Ichthyologists and Herpetologists, Spec Publ 1. Allen Press, Lawrence, KS, p 35-37

Puebla O (2009) Ecological speciation in marine versus

Editorial responsibility: Ivan Nagelkerken,

Adelaide, South Australia, Australia freshwater fishes. J Fish Biol 75:960-996

Rohlf JF (2010a) tpsUtil, version 1.44. Department of Ecology and Evolution, State University of New York at Stony Brook, Stony Brook, NY

Rohlf JF (2010b) tpsDig, version 2.14. Department of Ecology and Evolution, State University of New York at Stony Brook, Stony Brook, NY

Rohlf JF (2010c) tpsRelw, version 1.49. Department of Ecology and Evolution, State University of New York at Stony Brook, Stony Brook, NY

Schiemer L, Niedermüller S, Herler J (2009) The influence of colony size and coral health on the occupation of coralassociated gobies (Pisces: Gobiidae). Coral Reefs 28: $137-142$

Sebens KP (1987) The ecology of indeterminate growth in animals. Ann Rev Ecol Syst 18:371-407

Untersteggaber L, Mitteröcker P, Herler J (2014) Coral architecture affects the habitat choice and form of associated gobiid fishes. Mar Biol 161:521-530

> Vytopil E, Willis BL (2001) Epifaunal community structure in Acropora spp. (Scleractinia) on the Great Barrier Reef: implications of coral morphology and habitat complexity. Coral Reefs 20:281-288

Wall M, Herler J (2009) Postsettlement movement patterns and homing in a coral-associated fish. Behav Ecol 20: 87-95

Webb PW (1983) Speed, acceleration and manoeuverability of two teleost fishes. J Exp Biol 102:115-122

Webb PW (1984) Body form, locomotion and foraging in aquatic vertebrates. Am Zool 24:107-120

Webb PW, LaLiberte GD, Schrank AJ (1996) Does body and fin affect the maneuverability of fish traversing vertical and horizontal slits? Environ Biol Fishes 46:7-14

Wiederholm AM (1987) Habitat selection and interactions between three marine fish species (Gobiidae). Oikos 48: 28-32

Wilkinson CR (2004) Status of coral reefs of the world. Australian Institute of Marine Science, Townsville

Wong MYL, Buston PM, Munday PL, Jones GP (2007) The evolution of animal societies: The threat of punishment enforces peaceful cooperation and stabilizes queues in a coral-reef fish. Proc R Soc Lond 274:1093-1099

- Wong MYL, Munday PL, Buston PM, Jones GP (2008) Fasting or feasting in a fish social hierarchy. Curr Biol 18: R372-R373

Z Zelditch ML, Sheets DH, Fink WL (2000) Spatiotemporal reorganization of growth rates in the evolution of ontogeny. Evolution 54:1363-1371

Submitted: June 4, 2013; Accepted: December 10, 2013

Proofs received from author(s): February 22, 2014 